\title{
LOS PROCEDIMIENTOS EN EL APRENDIZAJE DE LA FÍSICA
}

\author{
SEVILLA SEGURA, $\mathrm{C}$. \\ IB Isabel de Villena, Valencia.
}

\section{SUMMARY}

After an analysis of the function of the learning procedures we can identify some noticeable intellectual activities: skills and strategies. By appealing to some concrete examples we can see its importance in the process of both the teaching/learning of Physics at pre-university levels.

\section{INTRODUCCIÓN}

La adaptación de los nuevos diseños curriculares abiertos y prescriptivos, a las organizaciones de centro y aula, supone para los profesores Ia realización de un trabajo nuevo, desconocido y cuyas características no parecen definidas con la claridad suficiente.

Los márgenes de libertad de acción que un currículo de esta naturaleza proporciona y la identificación de los procedimientos como parte constituyente de los conocimientos a programar son las principales novedades. En torno a ellas surgen la mayoría de las dificultades.

En este trabajo resumiremos algunas conclusiones deI análisis de los contenidos del currículo, de las relaciones entre los conocimientos conceptuales y los procedimientos, del papel y significado de éstos y de las consecuencias que se derivan para el aprendizaje de la física. Expondremos también las líneas directrices de un posible modelo didáctico, en el que se confiere a los procedimientos un tratamiento conjunto y similar al de los conceptos.

\section{LOS PROCESOS MEDIADORES EN EL APRENDIZAJE}

En términos generales entendemos el aprendizaje como un proceso de aproximación desde la estructura cognitiva del que aprende hasta el campo de conocimientos a los que pretende acceder. En el aprendizaje escolar se intenta acelerar este proceso a través de la planificación.
Planificar la acción didáctica implicará no sólo explicitar el punto de vista desde el que se hace la propuesta concreta y se interpretan los resultados obtenidos, sino también, identificar aquellas variables significativas cuya modificación será considerada indicador de aprendizaje y proponer los procedimientos más adecuados para la transformación.

Desde nuestra perspectiva teórica podemos seleccionar Ias siguientes variables relevantes, esquematizando el proceso como sigue en Ia tabla I.

La misma idea de proceso ya sugiere la necesidad de mediadores entendidos como elementos de apoyo que facilitan la realización y comprensión de una transformación intrínsecamente compleja.

El análisis del desarrollo observado en alumnos que aprenden significativamente nos ha permitido identificar algunas actividades mentales, que son parte de los procedimientos, a las que denominamos destrezas y estrategias.

Denominamos destreza la aptitud, pericia o habilidad para desempeñar una acción individual específica. Algunas destrezas comunes son: comparar, clasificar, observar, informar, relacionar, definir, codificar, analizar, interpretar, inferir, deducir, establecer analogías.

Denominamos estrategia a los procesos mentales complejos, que incluyen destrezas y conceptos, y cuya finalidad es encontrar solución a una situación problema. No 
entendemos que las estrategias sean una mera yuxtaposición de destrezas y conceptos, sino más bien que, al articularse unos y otras en la resolución de un problema, adquieren una nueva dimensión que modifica su significado, sus relaciones anteriores, dando lugar a procesos creativos y al planteamiento de nuevas situaciones problema.

No existe una separación clara entre destrezas o entre éstas y las estrategias. Tampoco entendemos que la destreza se refiera a acciones más simples. Como actividades que encuentran su significado en procesos complejos, existen relaciones entre ellas que evolucionan dependiendo del contexto. Así, por ejemplo, interpretar implica atribuir significados, para lo cual hay que establecer relaciones con un marco de referencia previamente seleccionado. Relacionar es encontrar nexos de unión entre ideas. Estas conexiones abarcan desde las relaciones directas, de causa-efecto, que un niño podría establecer, hasta encontrar una ley empírica, en cuyo caso habría que realizar un análisis de factores y control de variables. Una de las manifestaciones más evolucionadas del establecimiento de relaciones lo constituye el concepto de función matemática. Pero la interpretación puede łimitarse a atribuir significados a un término seleccionando entre las distintas acepciones que figuran en el diccionario, o también puede referirse al significado de un concepto en una teoría (concepto de masa en las mecánicas clásica y relativista). La interpretación que realiza un alumno de un concepto viene determinada por su representación mental, por el modelo que el propio alumno ha ido desarrollando y que debería evolucionar a lo largo de su formación académica, en la medida en que lo haga el modelo de referencia, produciéndose de esta forma los necesarios cambios conceptuales.

También hemos denominado destreza a cada una de las tres modalidades del razonamiento por inferencia: Ia deducción, la inducción y la analogía, piezas clave del pensamiento racional.
Por oposición a las conclusiones obtenidas por deducción, que son necesarias, las que proceden de la inducción son probabilísticas y amplían el conocimiento de las premisas en Iugar de explicitarlo. Esto hace que los procesos inductivos parezcan destinados a la formulación de hipótesis de las que pueden extraerse consecuencias deductivas que permiten confrontarlas con los datos y mantenerlas mientras no sean falsadas.

Otro aspecto importante en el proceso de aprendizaje lo constituye el establecimiento de analogías, entendidas como nexos con algo conocido anteriormente. Sin el recurso a la analogía, cada proceso de aprendizaje implicaría partir de cero. Conviene precisar el significado del término para así entender mejor su función en el aprendizaje escolar.

Bunge (1969) se refiere al razonamiento analógico como un tipo particular de inferencia que permite el paso de un conjunto de proposiciones a otro. Distingue entre analogía sustantiva, cuando la relación de semejanza se establece a nivel de componentes, y analogía estructural, cuando la semejanza se refiere a la forma; es decir, cuando la semejanza se basa en que ambos conjuntos de proposiciones están regidos por el mismo tipo de leyes.

Encontramos ejemplos de todos los tipos de analogía en nuestras clases. Cuando un alumno ha aprendido a ajustar, según un método determinado, una reacción química de oxidación-reducción y lo aplica a los demás casos, está desarrollando un razonamiento analogico basado en la semejanza. Sin embargo, cuando aplica el análisis vectorial que aprendió en la resolución de problemas de dinámica a los de campo eléctrico, está realizando una analogía estructural, de mayor alcance que la anterior y más fructífera.

Hemos intentado mostrar, en una primera aproximación, que no existe una línea de demarcación nítida entre destrezas y estrategias y que la denominación responde

Tabla I

\begin{tabular}{|l|l|}
\hline \multicolumn{1}{|c|}{ Situación del alumno } & Disciplina objeto de estudio \\
\hline Explicaciones y conceptos previos poco relacionados entre sí. & Redes conceptuales complejas. \\
Nivel y complejidad de razonamiento que es capaz de realizar. & Razonamiento logico muy elaborado. \\
Explicaciones parciales referidas a situaciones concretas. & Mayor capacidad explicativa. \\
$\begin{array}{l}\text { Limitación a la parcela de realidad que conocen por } \\
\text { experiencia directa. }\end{array}$ & Superior nivel de abstraccion y generalización. \\
\hline
\end{tabular}


a la necesidad de análisis de los caminos a través de los cuales la mente del alumno evoluciona, aunque para ello, en ocasiones, la simplificación es obligada.

El análisis, desde una perspectiva de aprendizaje constructivista dinámico, de la forma en que aprenden nuestros alumnos (y de las dificultades de los que no lo logran) nos ha facilitado la posibilidad de establecer relaciones profundas entre conceptos y procedimientos, identificando éstos con actividades frecuentes en una clase de física y química, todo lo cual nos ha permitido no sólo comprender mejor algunas de las dificultades intrínsecas del aprendizaje, sino hacer propuestas didácticas más fundamentadas.

\section{DESTREZAS Y ESTRATEGIAS ENLA CLASE DE FISICA}

Una de las mayores dificultades que encuentra un profesor, tanto en la programación como en la interpretación de los fracasos de los alumnos frente a determinadas tareas, tiene su origen en la especificidad e individualidad del método utilizado por cada uno de ellos, que to convierte en una verdadera «caja negra». La posibilidad de identificar actividades intelectuales observables, relacionadas con los procedimientos que acompañan el aprendizaje de los conceptos, tienen una clara aplicación práctica.

En el proceso mental que realiza un alumno para aprender física, tanto si se refiere a adquisición de conceptos o resolución de problemas, teóricos o experimentales, aparecen toda una serie de destrezas y estrategias que permanecen enmascaradas y cuya elucidación nos parece clave para facilitar el aprendizaje significativo, la evaluación y el diagnóstico, imprescindibles en una correcta planificación didáctica.

A través de algunos casos concretos de actividades frecuentes en el proceso de enseñanza/aprendizaje de la física y química, intentaremos mostrar la función de destrezas y estrategias.

\section{Análisis de factores}

Uno de los ejemplos más claros de actividad intelectual, que se realiza con frecuencia en un curso de física es el análisis de factores.

Para resolver cualquier situación problema, ya sea teórica o experimental, utilizando un método de pensamiento racional, hay que recurrir casi siempre a realizar una tarea de análisis y selección de aspectos relevantes, estableciendo relaciones entre ellos y eliminando razonadamente aquéllos que no son significativos. Este análisis de factores se encuentra vinculado al esquema «manteniendo constante lo demás», sin el cual no resulta posible contrastar una relación mútiple $y$, por tanto, establecer las condiciones bajo las que se realiza una experiencia.
Los alumnos no recurren de forma espontánea a estas destrezas. Muchos alumnos, sobre todo en los niveles elementales, aun siendo capaces de enumerar los factores que afectan, por ejemplo, al valor de la resistencia de un conductor, cuando se les pide que establezcan las condiciones de contrastación experimental, no son capaces de explicar que deben mantener todas las variables con un valor fijo, menos aquélla cuya influencia se desea conocer.

Además de las dificultades en la planificación de experiencias, la falta de dominio de estas destrezas tiene repercusiones en la comprensión del significado de funciôn matemática (piénsese en la relación matemática a la que se puede llegar tras analizar los resultados de una experiencia) y en el complejo proceso de emisión de hipótesis por cuanto constituye un estadio preliminar del mismo. (Aunque en ocasiones se identifica análisis de factores con emisión de hipótesis, consideramos ésta una trivialización del significado de la hipótesis en física).

Muchas veces, los problemas que planteamos dan hecho el análisis de factores, aparecen como datos los valores de todas las variables de que depende la incógnita o se pueden calcular fácilmente. Con enunciados abiertos, como, por ejemplo, «un móvil que se desplaza por una superficie horizontal, cuando divisa un precipicio, ¿qué hace para no caerse?", se facilita la tarea al poner de manifiesto: $1^{\circ}$ ) la necesidad de elegir la variable determinante (no caerse implica aplicar una fuerza de frenado determinada o, lo que es equivalente, una aceleración de frenado); $2^{\circ}$ ) fijar las condiciones iniciales ( $y$ cuáles son intercambiables; por ejemplo, si fijamos la distancia de frenado y la velocidad inicial, no es necesario el tiempo de frenado); $3^{\circ}$ ) establecer las relaciones entre to conocido y lo que se pretende averiguar y las condiciones en que se cumplen.

Tanto en el planteamiento del trabajo experimental como en la resolución de problemas, es necesario realizar análisis previos. Es tarea del profesor facilitar el aprendizaje de esta destreza intelectual, colaborando con el alumno en el desarrollo de recursos que le permitan afrontar y resolver problemas cada vez más complejos.

\section{Analogía}

Como ya señalamos, en su aplicación didáctica, la analogía aparece como analogía sustantiva y como estructural. La analogía sustantiva, basada en la similitud de contenido, es la más común. En función de ella se agrupan los ejercicios o problemas del mismo tipo, referidos al mismo tema (por ejemplo, aplicaciones de la ley de Ohm a los circuitos de corriente continua, estequiometría de reacciones químicas en estado gaseoso) con la pretensión de afianzar conocimientos. El uso de esta analogía permite iniciar la actividad mental del alumno en razón de la proximidad temática.

Mayor interés presenta la analogía estructural, por cuanto permite generalizar conocimientos, al distinguir lo fundamentai de lo accidental . 
Un ejemplo común en física lo constituye la analogía estructural, basada en el análisis vectorial. Aunque el cálculo vectorial, como mecanismo de análisis de situaciones, ya se utiliza en cinemática, tanto en dinámica como en campo eléctrico, constituye uno de los escollos fundamentales con que se encuentran los alumnos a la hora de resolver problemas.

Cuando se plantea a los alumnos el primer problema de dinámica en que aparecen varias fuerzas, actuando en direcciones distintas (por ejemplo, plano inclinado con rozamiento...), se observa que la mayoría son capaces de identificar las fuerzas, incluso de calcular su módulo, pero no son capaces de llegar a la solución final. No son capaces de aplicar a esta nueva situación los conocimientos de stuma vectorial adquiridos en otro contexto. La misma situación aparece con problemas de ley de Coulomb y de campo eléctrico debido a distribuciones puntuales de carga. Los alumnos no recurren a la analogía de forma consciente. No establecen relación entre estas dos situaciones, muy probablemente porque no hacen abstracción de lo que constituyen rasgos fundamentales de la situación, que son los que permiten aplicar el cálculo vectorial.

\section{La explicación}

Entendemos la explicación como una comparación entre un modelo teórico (estructura lógico-matemática entre proposiciones) y una situación física real, con la intención de establecer relaciones que permitan sacar conclusiones. La explicación constituye una tarea básica de cualquier planteamiento científico, razón suficiente por la que resulta interesante desarrollar esta estrategia entre nuestros alumnos, tanto más cuanto que los alumnos confunden, con frecuencia, la descripción de un fenómeno con su explicación.

Nos referiremos al tiro horizontal, planteado generalmente como la explicación de un nuevo movimiento, a la Iuz de los conceptos teóricos adquiridos (cinemática de los movimientos rectilíneos uniformes y uniformemente acelerados). La explicación incluye su contrastación experimental, todo lo cual involucrará una serie de destrezas y estrategias, que generalmente se mantienen ocultas y que sólo utilizan algunos alumnos, especialmente lúcidos, pero sin ser capaces de explicitarlo.

Podemos plantear el problema de forma directa: encontrar una explicación a un nuevo movimiento, el tiro horizontal, partiendo de la observación de un lanzamiento real y teniendo en cuenta los conocimientos de cinemática adquiridos. Una primera discusión entre los alumnos, conduce a la idea de que no es un movimiento simple, asimilable a ninguno de los ya estudiados (MRU, MRUA, MCU, MCUA), sino un movimiento compuesto por uno horizontal, que es rectilíneo uniforme, y otro verticat, que es uniformemente acelerado y que ambos tienen lugar simultáneamente. La primera dificultad aparece aquí; los alumnos confunden el análisis del movimiento con la explicación propiamente dicha. La idea de un movimiento compuesto de otros dos, perfectamente de- finidos y simultáneos ya les parece, a muchos alumnos, una explicación suficiente.

La estrategia que conduce a la resolución del problema, incluye varias destrezas:

a) Codificar, en términos matemáticos, Ia idea de movimiento compuesto, que incluye no sólo escribir las ecuaciones correspondientes sino también tener en cuenta su carácter vectorial y relacionar ambos movimientos, recurriendo al principio de superposición (suma vectorial) y simultaneidad.

b) Realizar una observación detallada que conduzca a la idea de que Ia trayectoria del movimiento se ajusta a una rama de parábola.

c) Idear la forma de obtener experimentalmente valores significativos, que constituyen la prueba contrastable del supuesto inicial, en este caso puntos de la trayectoria.

d) Realizar un análisis de factores y disponer del esquema manteniendo constante lo demás, para establecer las condiciones bajo las que debe realizarse la experiencia (velocidad de lanzamiento constante en un punto en que la aceleración de la gravedad también lo es).

e) Interpretar los resultados experimentales (recurriendo a representaciones gráficas) y comparar con el supuesto inicial para sacar conclusiones.

El aprendizaje significativo del tiro horizontal, que implica, por una parte, ser capaz de explicar el nuevo movimiento y, por otra, generalizar los esquemas desarrollados hasta lograr aplicarlos correctamente a la resolución de situaciones análogas(barcos que cruzan róos con corriente, objetos que caen con viento, tiro oblicuo), aparece unido a toda una serie de destrezas y estrategias, sin las cuales no parece fácil seguir el proceso.

Hemos intentado mostrar, a través de algunos ejemplos, la cantidad de conocimientos procesales que acompañan a los aprendizajes significativos de los conceptos físicos incluidos en un curso de secundaria o bachillerato. Hemos intentado mostrar también cómo se dificulta el aprendizaje de conceptos y la construcción de métodos de trabajo racionalmente fundamentados si en el planteamiento didáctico no hacemos explícitos, al mismo nivel que los conceptos, los procedimientos.

\section{ELEMENTOS DE UN MODELO DIDÁCTICO}

La idea de procedimientos (destrezas y estrategias) asociados a los conceptos y mediadores del aprendizaje orienta un planteamiento didáctico en el que la capacidad intelectual del alumno no se acepta como una característica personal sino como una capacidad susceptible de desarrollarse en el proceso de aprendizaje.

Entendemos que plantearse problemas y desarrollar toda la actividad que puede conducir a su solución forma 
parte de la actividad intelectual más genuina. A lo largo de la historia, el conocimiento humano se ha construido siguiendo la pauta de buscar respuesta a los grandes problemas que la humanidad se ha ido planteando.

Los conocimientos que los alumnos deben adquirir durante el curso académico pueden tener su origen en la búsqueda de respuesta a diferentes cuestiones planteadas. La construcción de conocimientos conlleva, con frecuencia, la elaboración de modelos teóricos. Ello no es posible sin introducir conceptos y recurrir a destrezas y estrategias intelectuales.

En la propuesta didáctica sugerida consideramos los conceptos y procedimientos como instrumentos útiles en la tarea general de resolución de probiemas.

Desde nuestro punto de vista, se puede organizar la actividad de la clase en tres etapas, de forma que se facilite a los alumnos la tarea de aprendizaje:

- Plantear problemas que den origen a un marco genérico en el que el trabajo adquiere significado.

- Dotarles de instrumentos que les permitan la elaboración de concepciones teóricas.

- Que tomen conciencia de lo que hacen, reflexionando, revisando y criticando la tarea realizada. En definitiva, que reelaboren el proceso seguido una vez finalizado.

Así, en cada unidad didáctica se incluyen tres fases: planteamiento de problemas, introducción de conceptos y procedimientos intelectuales propios para la resolución y síntesis. Desarrollaremos algunas de las características y funciones de cada una de las fases que suponemos que constituyen la unidad didáctica.

\section{Planteamiento de problemas}

Entendemos que un problema constituye una dificultad cuya resolución no es inmediata, sino que requiere la elaboración de una estrategia. Lo que constituye un problema para una persona puede no constituirlo para otra.

En el aprendizaje de clase podemos distinguir dos situaciones cotidianas: el aprendizaje de informaciones, más o menos puntuales, que se realiza de forma memorística, y el aprendizaje de conceptos, que requiere un proceso más complejo en el que se establecen relaciones con otros conceptos, se interpretan significados. Tanto uno como otro se ven favorecidos cuando se realizan en contextos en los que el alumno percibe con facilidad que el nuevo aprendizaje tiene sentido, le encuentra utilidad, es decir, justifica el trabajo que realiza.

El planteamiento de problemas generales, que permite al alumno situarse en cada momento y coordinar sus acciones, proporciona, en nuestra opinión, un marco conveniente de aprendizaje.

La resolución de una situación problema general a veces obliga a realizar un análisis a partir del cual se planteen situaciones más específicas otras veces supondrá la observación de una situación experimental que aproxime el tema general al alumno; en cualquier caso, gozará de la flexibilidad necesaria para adaptarse al nivel y condiciones del grupo de alumnos a los que se dirige.

Las situaciones problema planteadas a los alumnos deberían cumplir dos requisitos elementales:

a) Lograr interesarlos, implicarios, promover su actividad.

b) Que el planteamiento del problema genere un esquema global en el que el alumno pueda insertar sus conocimientos.

\section{Introducción de conceptos y procedimientosintelectuales}

Una vez el alumno hace suyo el problema, procede a elaborar una estrategia de resolución. Para ello necesita disponer de conocimientos y procedimientos intelectuales. Los contenidos conceptuales y de procedimiento constituyen los instrumentos válidos para abordar la resolución del problema, directamente o a través de los subproblemas planteados. El análisis de un problema complejo para obtener a partir de él otros más fácilmente abordables también es una destreza.

El alumno recurre a un concepto, o a una operación intelectual (destreza), de acuerdo con la estrategia general, elaborada para resolver un problema, en la que el concepto o el procedimiento cumplen una función. Para ello evoca conocimientos previos o incluye las sugerencias del profesor.

En nuestra opción didáctica, lo más importante no es que el alumno llegue a respuestas correctas, en el menor tiempo posible, sino que recorra el camino que supone que ha de conducirle a la solución y sea capaz de detectar sus errores y deficiencias (de concepto, de razonamiento, de recursos), que tome conciencia de ellos y los supere.

En este proceso, el profesor debería proporcionarle los instrumentos necesarios, conceptuales y de procedimientos, procurando satisfacer la necesidad de cada cual en el momento oportuno. Propocionar la información «personalizada» no resulta tan diff́cil si se piensa en las posibilidades disponibles:

- Trabajo en grupo, que permite un intercambio de ideas entre los alumnos.

- Diálogo con el profesor, individual o de grupo, cuando la dificultad es colectiva. En estas ocasiones el profesor proporciona la información necesaria, replantea el problema, sugiere procedimientos...

- Búsqueda bibliográfica. En ocasiones suplida por una exposición oral del profesor.En el contexto académico (número de asignaturas, de horas semanales dedicadas a cada una, formación y dedicación de los alumnos) no es posible esperar que los alumnos «reconstruyan» todos 
los grandes temas de la historia de la ciencia. Resultaría una pretensión ingenua esperar que los alumnos propongan un enunciado de las leyes de Newton como solución a los problemas planteados.

En la misma línea, el profesor organiza la discusión de Ios conceptos previos, propone ejercicios para afianzar los conceptos fundamentales, selecciona problemas experimentales, marca el ritmo de trabajo general.

Un buen control del nivel de aprendizaje lo constituye la aplicación de los conceptos y estrategias aprendidos en la resolución de un problema a la solución de situaciones análogas nuevas. Por ejemplo, en los primeros niveles, despues de estudiar los MRU y MRUA, los alumnos deberían ser capaces de «resolver el problema» del movimiento bajo la acción de la fuerza peso.

\section{Síntesis}

La fase final consiste en la elaboración de una sintesis del tema. Los alumnos disponen de material disperso, que han ido utilizando de forma parcelada y que les ha

\section{REFERENCIAS BIBLIOGRÁFICAS}

AAVV, 1990. Propuesta de diseño curricular. (Generalitat Valenciana: Valencia).

ANTÚNEZ, S., DEL CARMEN, LL., IMBERNON, F, PARCERISA, A. y ZABALA, A., 1991. Delprojecte educatiu a la programació d'aula. (Graó: Barcelona).

BUNGE, M., 1969. La investigación científica. (Ariel: Barcelona).

COLL, C., POZO, J.I., SARABIA, B. y VALLS, E., 1992. Los contenidos en la Reforma. Enseñanza y aprendizaje de conceptos, procedimientos y actitudes. (Santillana: Madrid).

COLL, C., 1990. Aprendizaje escolar y construcción de conocimientos. (Laia: Barcelona).

GIMENO SACRISTÁN, J., 1981. Teoría de la enseñanza y desarrollo del currículo. (Anaya: Madrid).

HEINZE-FRY, J.A. y NOVAK, J.D., 1990. Concept mapping brings long-term movement toward meaningful learning, Science Education, 74(4), pp. 461-472.

INHELDER, B. y PIAGET, J., 1972. De la lógica del niño a la lógica del adolescente. (Paidos: Buenos Aires).

INHELDER, B.,1975. Aprendizaje yestructuras del conocimiento. (Morata: Madrid).

JOHSUA, S. y DUPIN, J.J., 1991. In physics class, exercises can also cause problems..., International Journal of Science Education, 13(3), pp. 291-301.

LANG DA SILVEIRA, F., MOREIRA, M.A. y AXT, R., 1992. Habilidad en preguntas conceptuales y en resolución de problemas de física, Enseñanza de las Ciencias, IO(1), pp. 58-62. permitido ir comprendiendo conceptos, descubriendo actividades intelectuales propias del pensamiento racional. En la síntesis deben figurar todos los conceptos y las relaciones que se establecen entre ellos; en cierta forma es lo que Novak (1989) llama mapa conceptual. En la síntesis escrita deberían figurar también los problemas resueltos, agrupados por analogía, todo ello redactado de forma clara y coherente.

La actividad desarrolłada al elaborar la síntesis supone una reorganización de conocimientos, una reflexión sobre el trabajo realizado y las dificultades superadas, que nos parece fundamental para consolidar el aprendizaje.

EI profesor se sorprende al descubrir las dificultades que tienen los alumnos para realizar esta labor de síntesis. Llama también la atención el paralelismo entre la calidad de la síntesis y la calificación obtenida en un ejercicio de examen.

En realidad el trabajo de síntesis es el que cualquier estudiante consciente realiza como preparación de un examen, pero frente al que los actuales alumnos se encuentran desorientados quizá por recurso excesivo a un método más escolar basado en la memorización.

LANGFORD, P., 1990. El desarrollo del pensamiento conceptual en la escuela secundaria. (Paidós: Barcelona).

MORENO, M. y SASTRE, G., 1983. Apréndizaje y desarrollo intelectual. (Gedisa: Méjico).

NOVAK, J.D. y GOWIN, A., 1989. Aprender a aprender. (Martínez Roca: Barcelona).

NOVAK, J.D., 1991. Ayudar a los alumnos a aprender. La opinion de un profesor-investigador, Enseñanzade las Ciencias, 9(3), pp. 215-228.

OMASTA, E. y LUNETTA, V.N., 1988. Exploring functions: A strategy for teaching physics concepts and problemsolving, Science Education, 72(5), pp. 623-636.

PAGIGLIONE, C. y TORRACA, E., 1990. Logical processes in experimental contexts and chemistry teaching, International Journal of Science Education, 12(2), pp. 187-194.

PIAGET, J., 1977. La explicación en las ciencias. (Martínez Roca: Barcelona).

POZO, I.1., 1987. Aprendizaje de la ciencia y pensamiento causal. (Visor: Madrid).

POZO, J.I., 1989. Teorias cognitivas del aprendizaje. (Morata: Madrid).

SEVILLA, C., VIÑA, R. y GARCÍA, P., 1993. Los procedimientos en el bachillerato. Aula de Innovación Educativa, 2(12), pp. 73-77.

WHEATLEY, G.H., 1991. Constructivist perspectives on science and mathematics learning, Science Education, $75(1)$, pp. 9-2I. 